\title{
Nonintrusive Fiber Monitoring of TDM Optical Networks
}

\author{
Bert De Mulder, Student Member, IEEE, Wei Chen, Student Member, IEEE, Johan Bauwelinck, Member, IEEE, \\ Jan Vandewege, Member, IEEE, and Xing-Zhi Qiu, Member, IEEE
}

\begin{abstract}
This paper introduces a new embedded nonintrusive fiber-monitoring technique for time-division-multiplexing optical networks. It allows an optical transmitter to characterize the fiber plant from reflections caused by data bursts transmitted across the network instead of dedicated test signals. The probing is performed with minimal burden on data traffic so that many measurements can be averaged to improve accuracy. The method is very suitable for embedded optical time-domain reflectometers (OTDR), which reuse a network node's optical data transmitter for OTDR excitations and embed a reflectometer inside the fiber endpoint. This paper models the OTDR with Laplace transforms, an approach previously unpursued, after which it is explained how reflections from multiple data bursts with arbitrary width can be converted into one normalized format. This new class of OTDR excites the fiber with a negative step of light instead of the conventional short pulse. The signal-to-noise ratio (SNR) for backscatter and Fresnel reflections caused by the negative step and pulse are compared theoretically. It is shown that negative-step OTDR breaks the tradeoff between excitation pulsewidth and distance resolution, has a natural separation between fiber backscatter and Fresnel reflectors, and improves the SNR of nonreflective events.
\end{abstract}

Index Terms-Fault detection, optical-fiber communication, optical-performance monitoring, optical time-domain reflectometry (OTDR), passive optical networks (PONs).

\section{INTRODUCTION}

$\mathbf{O}$ PTICAL network monitoring becomes critical to maintain tomorrow's optical-fiber networks, which persist in offering higher bandwidths and faster network services. Monitoring can pinpoint problems before they put a burden on network traffic, so countermeasures like protection and restoration can be taken preventatively, which decreases the operational expenses significantly, and can certify quality of service agreements. Several techniques are used to monitor the operational performance of optical networks [1]. Current practice often ends by verifying the correct transmission of data bits over the optical-fiber plant with, e.g., cyclic-redundancy checks, in agreement with [2]. Although several techniques allow to examine the physical layer in more detail, these are not widely deployed because of high implementation costs and negative impact on network operation and availability. Nevertheless, a fast preventive and continuous diagnosis of the integrity of the fiber network and a quick diagnosis on deteriorating link

Manuscript received May 8, 2006; revised September 27, 2006.

The authors are with the Department of Information Technology, INTEC/ IMEC, Ghent University, B-9000 Ghent, Belgium (e-mail: bert.demulder@ intec.ugent.be; wei.chen@intec.ugent.be; johan.bauwelinck@intec.ugent.be; jan.vandewege@intec.ugent.be; xingzhi@intec.ugent.be).

Digital Object Identifier 10.1109/JLT.2006.887184 performance are important assets in all kinds of performancecritical optical-fiber links and networks [3].

Optical time-domain reflectometry (OTDR) [4] including backscattering is a powerful method for defining the exact location and cause of performance degradation in an opticalfiber plant. OTDR can diagnose fiber breaks, splice losses, reflections, and also increasing distributed losses due to fiber aging, cable stress, or water penetration. Conventional OTDR instruments excite the fiber with an optical pulse and characterize the bearer from returning optical reflections, but the technology is too expensive if used only for fiber-plant monitoring. On the other hand, increasing deployment and complexity of optical-fiber plants and their use for critical applications create a need for inexpensive embedded OTDR units. When added to every consisting optical network element, fiber networks could be monitored with little impact on network operation.

Embedded OTDR measurements should not interfere with data traffic being transmitted along the optical fiber. This nonintrusive mode can be accomplished by using wavelengthdivision-multiplexing (WDM) techniques, injecting pulsed light of a different wavelength into the fiber [5]. But, WDM OTDR requires a dedicated transmitter (TX) and receiver (RX) at this second wavelength and an optical system separating signals at the different wavelengths. Moreover, the cost of the embedded OTDR unit must be low. A recent idea for passive optical-access networks (PON) is the embedded OTDR unit that reuses the laser driver and laser diode that are present at the fiber endpoint for data transmission [6], [7]. This is a good approach, as the measurement will show the attenuation in function of distance at the correct wavelength. The OTDR curve depends on the excitation wavelength and cannot be measured accurately by using a separate wavelength for OTDR. This system also reduces the cost to a certain extent, because no dedicated OTDR laser and driver are needed, but an additional coupler and photodetector are still required.

In time-domain-multiplexing (TDM) optical-networks time slots are reserved for each TX, which sends data in bursts instead of continuously. Similarly to OTDR pulses, data bursts cause reflections from the fiber plant, which can be used to derive the channel performance. This forms the basis for the presented nonintrusive OTDR technique assembling a detailed OTDR curve out of measurements on optical-echo signals associated with the data bursts the system is transporting. Every data burst transmitted can improve the knowledge of the channel-link status when after transmission an empty time slot is available for observing the optical echo. A practical problem is that in such system the OTDR has no control over 


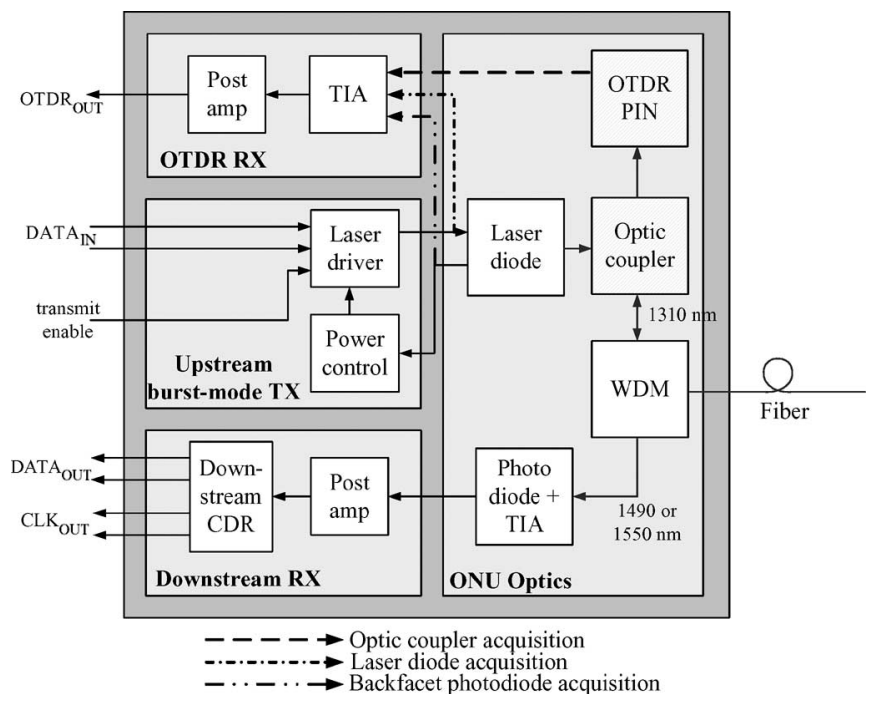

Fig. 1. Integration of OTDR functionality inside ONU. The shaded blocks from the optics subsystem are only required in case the OTDR echoes are sampled with an optic coupler. In case acquisition is done on the laser or backfacet photodiode, the OTDR does not need the OTDR PIN and optic coupler.

the length of the data burst. This paper explains how arbitrary burst reflections can be processed into a common format, which will be identified as the negative-step response. This novel way of fiber excitation is compared to the traditional approaches, and new modes of operation are identified.

The best contemporary example of optical TDM networks is PON [8], [9]. For PONs, the link budget is tight because the optical-network unit (ONU) is a very cost-critical element residing at the customer premises. Fig. 1 illustrates OTDR functionality integrated with the ONU at minimum cost. The data TX is reused to create OTDR excitation pulses, with the optical power that is compliant to the PON standard. In case of Gigabit PON (GPON), e.g., the launched average upstream power ranges between -2 and $3 \mathrm{dBm}$ for the upstream data [10]. As this excitation power is considerably lower than conventional instruments, the OTDR dynamic range is reduced.

Fig. 1 also presents three nonconventional low-cost methods to acquire optical echoes caused by the OTDR excitation. The first approach uses a 10/90 optic coupler instead of a conventional 50/50 splitter to separate reflections from transmitted light [6], [7], but this approach requires additional dedicated optics and reduces the link budget. In case the laser module contains no isolator, the echo can also be detected by using the laser diode as a photodetector [11], [12]. This approach is very economical but requires fast switching of the laser diode between the laser driver and the sensitive OTDR RX. A similar switchover mechanism occurs when measuring echoes on the backfacet photodiode connected to the laser [12]. All methods have a similar aggregate responsivity, which is a fraction of the responsivity of a PIN photodetector. The reported responsivity for a vertical-cavity surface-emitting laser (VCSEL) is of $0.2 \mathrm{~A} / \mathrm{W}$ [11], although much below the quantum limit, is even optimistic in many cases. Because this, again, reduces the signal-to-noise ratio (SNR) of the detected reflections,

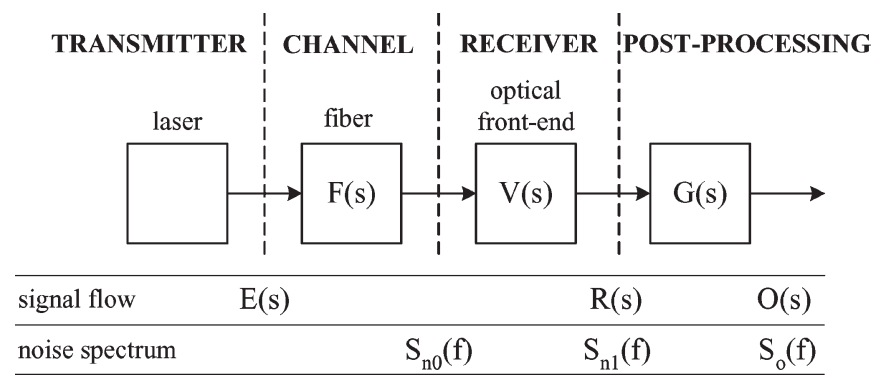

Fig. 2. Simplified model of generic OTDR systems.

other procedures are needed to improve OTDR measurement accuracy, which are presented in this paper.

In Section II, a model of the OTDR system will be derived using representations with Laplace transforms instead of the traditional time-domain models. The mathematical model will be used in Section III to explain the concept of nonintrusive fiber monitoring, which uses the reflections of data burst to characterize the fiber instead of exciting with dedicated OTDR pulses, and the concept of negative-step excitation is introduced. Subsequently, in Sections IV and V, an analysis is made of the SNR for classic-pulse response and the negativestep response, respectively, and both methods are compared. In Section VI, the negative-step response is illustrated with some simulations, showing the different nature of the occurring reflective and nonreflective events, and the benefits for negativestep OTDR are summarized in Section VII. Finally, a conclusion is given in Section VIII.

\section{MODEL OF OTDR S YSTEM}

Independent of the OTDR implementation, the system always consists of the same building blocks as illustrated in Fig. 2. The optical TX creates an optical excitation with Laplace transform $E(s)$ that is injected into the fiber system under test which reflects $E(s)$ due to imperfections with reflectivity $F(s)$. The reflected optical signal is acquired and conditioned by the OTDR RX with an overall RX transfer function $V(s)$. After digitization, further postprocessing may be required, which is presented with the function $G(s)$, containing, for example, further digital filtering. All blocks are assumed linear and timeinvariant.

This section models the complete OTDR. Conventional theoretical descriptions characterize the system in the time domain, but in this paper, the one-sided Laplace transform is preferred to derive the transients caused by causal injected pulses. The Laplace transform of a signal is written with a capital letter like $Q(s)$ while the time-domain transient is written as $q(t)$. All OTDR signals are assumed to be causal, which is the case if the excitation function is causal too. The Fourier transform $Q(f)$ is used rather than the Laplace transform $Q(s)$ when the influence of a linear-transfer function on noise is investigated. For a signal with finite energy, such as pulse reflections, the first can be derived from the latter by evaluating the Laplace transform along the imaginary axis

$$
Q(f)=\left.Q(s)\right|_{s=j 2 \pi f} .
$$


As both Laplace transform and Fourier transform are denoted with the same capital letter, distinction is made by their dependence on the frequency $f$ response on the Laplace parameter $s$. This formality will be kept throughout this paper.

\section{A. Excitation Signal}

The optical TX is typically a semiconductor laser diode [4], [11], [13]. In PONs, most of the time, inexpensive isolatorless Fabry-Pérot lasers are preferred, which is fit for echo acquisition on the laser diode and backfacet photodiode. In conventional OTDR instruments, the excitation signal $e(t)$ is a causal pulse with peak power $P_{0}$ starting at $t=0$ having finite height $P_{0}$ and width $W$

$$
\begin{aligned}
p\left(T_{1}, T_{2}\right) & = \begin{cases}1, & \text { for } T_{1} \leq t \leq T_{2} \\
0, & \text { otherwise }\end{cases} \\
e(t) & =P_{0} p(0, W) .
\end{aligned}
$$

The one-sided Laplace transform of the pulse $e(t)$, with infinite rise and fall times, equals

$$
E(s)=P(s)=P_{0} \frac{\left(1-e^{-s W}\right)}{s} .
$$

Evaluating the magnitude of $P(s)$ along the imaginary axis yields the familiar sinc response.

In real systems, rise and fall times of the launched pulse will not be infinite but depend on the bandwidth of the optical TX. Given an OTDR RX with a few megahertz bandwidth and the electronics driving a laser diode at gigabit speed, this effect can be ignored.

\section{B. Fiber System}

The fiber impulse response $f(t)$ of the reflection channel with Laplace transform $F(s)$ consists of a few different contributors.

1) Fiber Backscatter: When an optical pulse travels through a fiber, diffuse light is reflected back toward the pulse source [14]. There are different reflection mechanisms, depending on the wavelength of the reflected light. At low excitation powers, Rayleigh scattering is dominant and the reflected light has the same wavelength as the incident light. The impulse response due to backscattering by an infinite fiber equals [15]

$$
f_{\mathrm{bs}}(t)=\eta e^{-\beta \nu t}
$$

where $\nu$ is the group velocity (in kilometers per second), $\beta$ the attenuation coefficient (in nepers per kilometer), and $\eta$ the backscatter parameter (in watts per joule). When the attenuation coefficient $\beta$ or the backscatter parameter $\eta$ is not constant along the fiber but depends on the distance from the optical TX, the simplified model of (5) is no longer sufficient. An increased attenuation constant could be caused by an over-bent fiber, causing light to couple out of the fiber, and temperature variations or acoustic vibrations may change the backscatter parameter $\eta$ along the fiber.

The impulse response of a first-order system is described by (5); hence, the Laplace transform of the reflection system equals

$$
F_{\mathrm{bs}}(s)=\frac{\eta \tau_{\mathrm{bs}}}{1+s \tau_{\mathrm{bs}}}, \quad \text { with } \tau_{\mathrm{bs}}=1 / \beta \nu .
$$

Typical values for the backscatter time constant $\tau_{\text {bs }}$ are in the order of a few tens of microseconds.

In case the fiber has a finite length $L$, the backscatter impulse response is time-constrained and equals to zero after $t_{\mathrm{st}}=$ $2 L / \nu$

$$
f_{\mathrm{bs}}(t)=p\left(0, t_{\mathrm{st}}\right) \eta e^{-t / \tau_{\mathrm{bs}}}
$$

with Laplace transform

$$
F_{\mathrm{bs}}(s)=\frac{\eta \tau_{\mathrm{bs}}}{1+s \tau_{\mathrm{bs}}}\left(1-e^{-t_{\mathrm{st}} / \tau_{\mathrm{bs}}} e^{-s t_{\mathrm{st}}}\right) .
$$

An important factor for finite-fiber sections is the attenuation constant $f_{\text {att }}$

$$
f_{\mathrm{att}}=e^{-t_{\mathrm{st}} / \tau_{\mathrm{bs}}}=e^{-2 \beta L} .
$$

This factor contains information about the two-way loss and can be used as a scaling factor for optical reflections that are protruding from after the first fiber section.

2) Fresnel Reflections: Fresnel reflections are caused by abrupt changes of the propagation medium. For example, when two fibers are joined with connectors, a small air slit always remains in between the fiber ends and causes a discrete localized reflection at that point. If this point is located at distance $D$ from the optical TX, the reflection equals [15]

$$
f_{\mathrm{fr}}(t)=R e^{-2 \beta D} \delta(t-2 D / \nu) .
$$

This represents a delayed delta impulse that was attenuated by the fiber section with length $D$. The unitless reflection coefficient $R$ defines the size of the reflection and depends on physical properties like the fiber cross section, the excited propagation modes, the geometry of the fiber ends and the gap, and the refraction index of the fiber and the gap-filling material.

The Laplace transform of impulse response (10) equals

$$
F_{\text {fr }}(s)=R e^{-2 \beta D} e^{-s \tau_{\text {fr }}}, \quad \text { with } \tau_{\text {fr }}=2 D / \nu .
$$

The magnitude of this reflection is independent on frequency and its phase is linear, which implies a fixed delay. This is because dispersion can be neglected at megahertz bandwidths.

3) Total Fiber Reflection: Using superposition, the aggregate reflection can be found by summing different contributors described by (8) and (11), which possibly need to be scaled with splice losses or the attenuation factor from (9) and delayed by preceding fibers' traveling time.

\section{Analog Front End}

A detector with responsivity $R_{p}$ (in amperes per watt) converts the optical reflection signal into a proportional photocurrent. The responsivity also involves the coupling factor from the optical coupler. A transimpedance amplifier (TIA) or highimpedance amplifier converts this photocurrent into an output voltage, with frequency-dependent transimpedance $R_{t}(s)$. During this process, it is assumed that a Gaussian noise current is present at the input of the photodetector, but likely, the noise spectrum is not constant with frequency. Using $R_{p}$, the current 
noise can be input referred as optical noise $n 0(t)$ with noise spectrum $S_{n 0}(f)$.

The TIA, which is the dominant contributor to the outputreferred voltage noise, has a noise spectrum that rises proportionally with frequency within the amplifier bandwidth [16] and becomes constant afterward. Therefore, a lot of noise resides above the TIA cutoff frequency and some reduction of noise can be obtained by cascading the TIA with a low-pass filter with transfer function $H(s)$. The order and type of this filter are a tradeoff between noise suppression and fast-transient settling.

Following amplifiers increase the signal level and map the backscatter system dynamic range to the input of an analog to digital (A/D) converter. In case that the amplifiers have adequate bandwidth and inject no substantial noise into the system, both signal and noise are multiplied by the gain $G$, resulting in an output signal $r(t)$ with noise $n 1(t)$. The analog front end is a linear system $V(s)$

$$
V(s)=R_{p} R_{t}(s) H(s) G .
$$

The output noise is defined as

$$
S_{n 1}(f)=S_{n 0}(f)|V(f)|^{2} .
$$

The filter shapes the noise spectrum and, therefore, also the total integrated noise, while keeping most of the useful signal information

$$
N 1=\int_{-\infty}^{+\infty}|V(f)|^{2} S_{n 0}(f) d f .
$$

The SNR of the signal after filtering is defined by the responsivity, transimpedance, the filter, and of course, the input-referred amplifier noise, so a careful design of the analog front end is needed to obtain a large dynamic range.

\section{System Identification}

If it would be possible to excite the fiber linearly with an optical delta impulse with an energy of $1 \mathrm{~J}$, the excitation function $E(s)$ would be identically one, and the system response would be equal to

$$
R(s)=F(s) V(s)=\hat{F}(s) .
$$

The measured response is the time-domain convolution of the fiber response and the RX response introduced by [17] as the smoothed fiber response.

In practice, launching a light impulse with zero length and infinite power is impossible. For any coherent light source, both peak power $P_{0}$ and the minimal pulsewidth $W$ are limited. The energy a realistic pulse can contain is then much smaller than $1 \mathrm{~J}$, resulting in weaker reflections and a decreased SNR. Conventional OTDR instruments measure the following reflection:

$$
R(s)=\underbrace{P_{0} \frac{\left(1-e^{-s W}\right)}{s}}_{P(s)} \hat{F}(s) .
$$

In case $W$ is small, the pulse spectrum can be approximated as $P_{0} W$ within the $\mathrm{RX}$ bandwidth. This results in the fiber being excited with an impulse with signal energy $P_{0} W$. When the peak source power $P_{0}$ is limited, the pulse energy can only be increased by applying a broader pulse. As the Fresnel reflection also has pulsewidth $W$, this makes it harder to locate the reflecting artifact. This tradeoff between pulsewidth and spatial resolution is a major restriction of conventional pulse OTDR [18].

Linear-circuit theory learns that there are other solutions for characterizing a system than measuring the impulse or pulse response, such as exciting the system with a unity step $P_{0} u(t)$

$$
u(t)= \begin{cases}0, & \text { for } t<0 \\ 1, & \text { for } t>0 \\ 1 / 2, & \text { for } t=0\end{cases}
$$

The impulse response can be calculated by deriving the step response with respect to $t$, and the step response can be found by integrating the impulse response between limits zero and $t$. The Laplace transform of the unity step is $1 / s$, so the reflection signal becomes

$$
R(s)=\frac{P_{0}}{s} \hat{F}(s) .
$$

Using the step response for system identification in OTDR can be very cumbersome. The reason is the limited isolation between OTDR TX and RX. This blinds the RX during transmission and makes it impossible to measure small reflections accurately. An alternative to the step excitation is the negativestep excitation $P_{0} \tilde{u}(t)$, which consists of excitation with power $P_{0}$ for a very long time and shutting it down suddenly

$$
\tilde{u}(t)= \begin{cases}1, & \text { for } t<0 \\ 0, & \text { for } t>0 \\ 1 / 2, & \text { for } t=0\end{cases}
$$

This can be rewritten as

$$
\tilde{u}(t)=1-u(t)
$$

which proves that the derivative with respect to $t$ of the system response to $-\tilde{u}(t)$ once again is equal to the impulse response of the system, which proves the validity of negative-step excitation.

One important remark is that $\tilde{u}(t)$ is not causal, so the onesided Laplace transform no longer can be used to define the transient of $r(t)$ after $t=0$. From (20), however, it follows that the response is equal to a dc response minus a step response. The response for $t>0$, thus, can be found by subtracting the step response from a step function of the size of the dc response. The Laplace transform of the reflection for $t>0$ can, therefore, be written as

$$
R(s)=P_{0} \frac{\hat{F}(0)-\hat{F}(s)}{s} .
$$

Exciting the system with a negative step is a novel approach to OTDR that can be superior to the classic excitation with a pulse. In the following paragraphs, the negative-step RX will be 

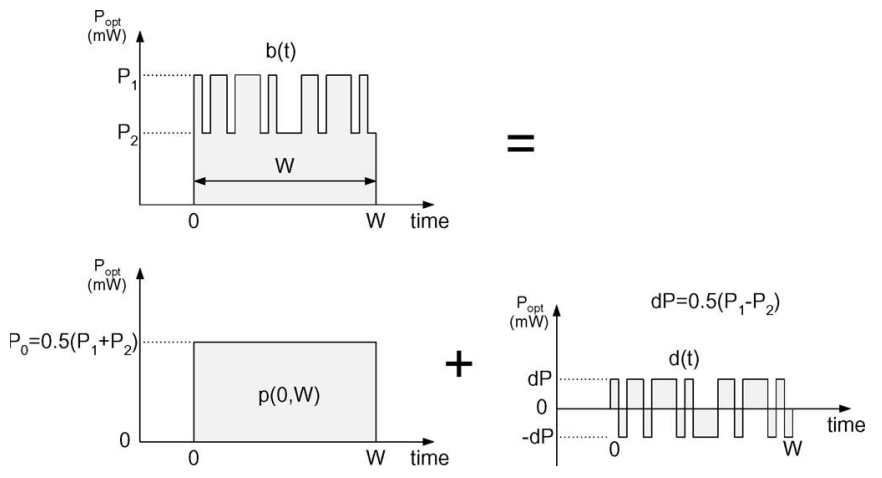

Fig. 3. Dissection of a data burst, which consists of a pulse $p(t)$ and a data signal $d(t)$.

analyzed and its performance compared to conventional pulse OTDR units.

\section{NONINTRUsive Fiber MONitoring}

In an operative TDM burst-mode network, any fiber endpoint transfers data bursts from time to time along the fiber. Of course these data burst also cause reflections along the communication channel. If a system could measure these reflections and derive channel characteristics from here, this would result in a true nonintrusive OTDR system. The problem that arises is that there is no control over the burst length $W$, while the excitation pulse for an off-the-shelf OTDR instrument has a controlled but fixed width.

\section{A. Data Burst}

Suppose the laser sends out a data burst $b(t)$, with a length $W$, but modulated at a high bit rate with useful data. The burst can have a return to zero or nonreturn to zero format, so in general, transmits the optical power $P_{1}$ when transmitting a "1," and $P_{2}$ when transmitting a " 0 ," where it is assumed that $P_{1}>P_{2}$. With

$$
\begin{gathered}
P_{0}=\frac{P_{1}+P_{2}}{2} \\
d P=\frac{P_{1}-P_{2}}{2} .
\end{gathered}
$$

The data burst can be written as

$$
b(t)=p(0, W)(P_{0}+\underbrace{d P x(t)}_{d(t)})
$$

which is illustrated in Fig. 3.

The infinite-random data bit stream $x(t)$ swings between minus and plus one with data period $T_{b}$. The burst consists of a pulse with height $P_{0}$ and a zero-mean random-data stream with magnitude $d P$. Random binary data is a sequence of bits in which the probability that any given bit in the sequence has a value of one or zero is independent of the value of all other bits in the sequence. If the data is fully random, there is no correlation between two consecutive data bits.
As the transmitted data is deterministic, this may not always be accurate. The transmitted burst, however, is known, so if it, for example, contains a lot of consecutive ones or zeros, the burst reflection can be neglected to avoid deterministic measurement errors.

The pulse response from the fiber was derived in the previous section, the influence of the data modulation is not clarified yet. The random-data model is used to define the frequency content of the data burst

$$
d(t)=d P p(0, W) x(t) .
$$

The autocorrelation function of an infinite random bit stream equals

$$
R_{x x}(\tau)=\lim _{T \rightarrow \infty} \frac{1}{2 T} \int_{-T}^{+T} x(t) x(t-\tau) d t .
$$

It is important to notice that there is no correlation between different bits, so the integration in (26) can be limited between $-T_{b}$ and $T_{b}$

$$
R_{x x}(\tau)=\frac{1}{2 T_{b}} \int_{-T_{b}}^{+T_{b}} x(t) x(t-\tau) d t .
$$

Under the condition that the data signal has infinite rise and fall times, the autocorrelation is a triangle function that starts at zero for $\tau=-T_{b}$, rises linearly to one for $\tau=0$, and then, drops linearly to zero at $\tau=+T_{b}$.

The power spectrum can be obtained by taking the Fourier transform of the autocorrelation, this results in

$$
X(f)=T_{b} \operatorname{sinc}^{2}\left(T_{b} f\right) .
$$

This can be considered as a noise spectrum. Because the signal swings between $+d P$ and $-d P$, this spectrum should be multiplied with $d P^{2}$, resulting in the modulation noise spectrum. The RX transfer function $V(s)$ will shape the modulation noise spectrum. Because we assumed the data period $T_{b}$ to be very small within the RX bandwidth, the spectrum is approximately constant with frequency and equal to

$$
d P^{2} T_{b} \text {. }
$$

The total noise now can be written as

$$
N_{\text {mod }}=\int_{-\infty}^{+\infty} d P^{2} T_{b}|V(f)|^{2} d f .
$$

This formula for the modulation noise is valid for the excitation signal. In practical cases, the bandwidth will be limited drastically. In case the reflection system is a Fresnel reflector as described by (11), the overall bandwidth is defined by the OTDR RX. Assuming $V(f)$ to be first order with cutoff frequency $f_{c}$, the integrated optical noise becomes

$$
N_{\text {mod }}=\frac{d P^{2} T_{b} f_{c}}{2}|V(0)|^{2}
$$


Next to the modulation noise, the burst of (24) also consists of a pulse with peak power $P_{0}$. The instant power is equal to

$$
S=P_{0}^{2}|V(0)|^{2} .
$$

The SNR is calculated from (31) and (32) as

$$
\mathrm{SNR} \cong \frac{P_{0}^{2}}{d P^{2}} \frac{2}{T_{b} f_{c}} .
$$

In case $P_{1} \gg P_{2}$, the first factor approaches unity. The SNR is inversely proportional to $f_{c}$. This can be understood when considering the response from a Fresnel reflector to a data burst. The smaller the bandwidth of the RX, the smaller the residual ripple caused by the modulation will remain after detection of the modulation noise. The SNR is also inversely proportional to $T_{b}$. The lower $T_{b}$, the more modulation noise is pushed toward higher frequencies and filtered out by the RX bandwidth. If the $\mathrm{RX}$ bandwidth is $5 \mathrm{MHz}$ and the data bit rate $1.25 \mathrm{~Gb} / \mathrm{s}$ like upstream GPON, this results in an optical SNR of $24.0 \mathrm{~dB}$. In conclusion, exciting the fiber system with a data modulated pulse results in the sum of a pulse response and modulation noise with power that is proportional to the signal. In fact, the ratio between the bit rate $1 / T_{b}$ and the $\mathrm{RX}$ bandwidth ratio defines the SNR.

In case the fiber reflection is backscatter, this is the process that imposes the dominant limit on the system bandwidth. The modulation noise inside the reflection signal will then be reduced even further. For example, in case the backscatter time constant equals $60 \mu \mathrm{s}$, the received optical SNR due to modulation noise improves to $40.7 \mathrm{~dB}$. As in most cases, wellinstalled fiber networks reflect backscatter predominantly; it shows that good reflection measurements can still be made despite the modulation noise.

\section{B. Conversion to the Negative-Step Response}

Suppose a burst is transmitted with length $W$ and injected into the fiber. We ignore the modulation noise. The RX response to the optical echo equals

$$
R(s)=P_{0} \frac{\left(1-e^{-s W}\right)}{s} \hat{F}(s) .
$$

The fiber reflection function $\hat{F}(s)$ is what should be derived from the pulse response. Rewriting (34) yields

$$
\hat{F}(s) \frac{P_{0}}{s}=\frac{R(s)}{1-e^{-s W}} .
$$

The left part of this equation can be interpreted as the smoothed step response of the fiber system. The last factor can be rewritten as an infinite sum

$$
\hat{F}(s) \frac{P_{0}}{s}=R(s) \sum_{k=0}^{+\infty} e^{-s k W} .
$$

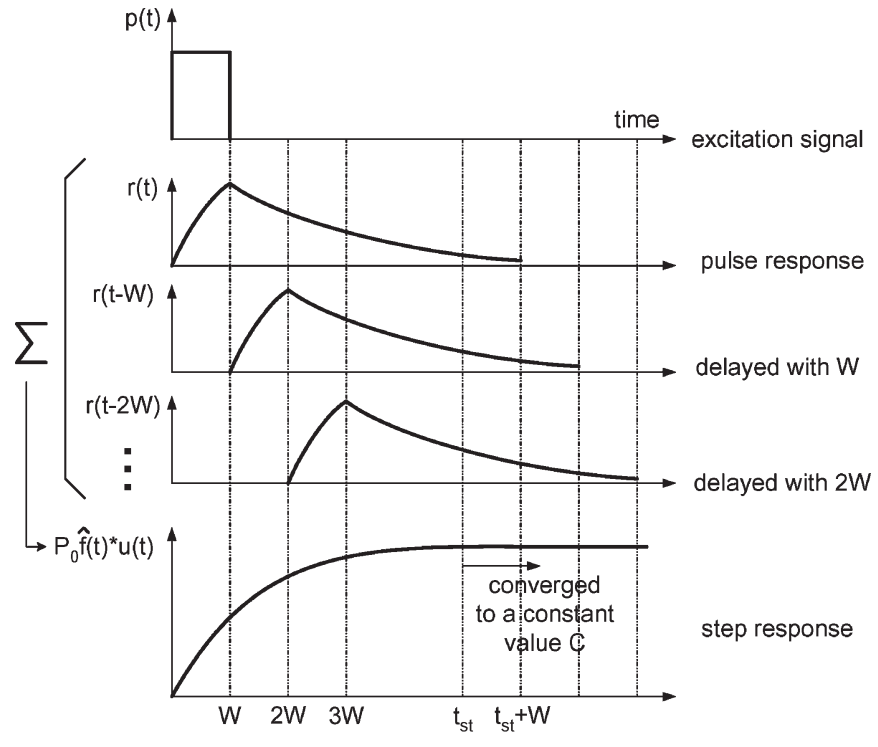

Fig. 4. Illustration of the transformation of the pulse response into the step response. The step response converges to a constant value $C$ after $t_{\mathrm{st}}$ in case the fiber has finite length. Therefore, the infinite sum from (37) can be reduced to a fixed number $K$.

In the time domain, this corresponds to

$$
P_{0} \hat{f}(t) * u(t)=\sum_{k=0}^{+\infty} r(t-k W) .
$$

This equation is illustrated in Fig. 4. It shows that the step response converges to a constant value $C$, which is independent of $W$ and equals

$$
C=\lim _{t \rightarrow+\infty} \hat{f}(t) * u(t) .
$$

Equation (37) proves that the smoothed step response can be derived from any pulse response with arbitrary width $W$ by time shifting the measured pulse response and summing them all. As impulse responses coming from an optical fiber are always time-limited to $t_{\mathrm{st}}$, the sum is limited to the biggest integer $K$ below $t_{\mathrm{st}} / W$. If $W$ is bigger than $t_{\mathrm{st}}$, no summing is needed and we directly calculate the step response of the system.

One important remark is that for realistic OTDR implementations it is impossible to make an accurate measurement when the laser is transmitting, like during the duration of the pulse. This means that the first part of the response from $t=0$ until $t=W$ cannot be measured

$$
\widehat{r}(t)= \begin{cases}0, & \text { for } 0 \leq t \leq W \\ r(t), & \text { for } t>W\end{cases}
$$

The function $w(t)$ is defined as the periodically extended but unknown first part of the $r(t)$ between $t=0$ and $t=W$

$$
w(t)=\sum_{k=0}^{+\infty}[r(t-k W)-\widehat{r}(t-k W)] .
$$


Equation (37) can now be rewritten as an infinite sum of timeshifted versions of $\widehat{r}(t)$, plus the periodically extended first part of the $r(t)$

$$
P_{0} \hat{f}(t) * u(t)=\sum_{k=0}^{+\infty} \widehat{r}(t-k W)+w(t) .
$$

Because the reconstructed step response converges to $C$ for $t \rightarrow$ $+\infty$, this periodic function can be derived from the infinite sum itself by time shifting the infinite sum of $\widehat{r}(t)$ back in time. The periodic residual $w(t)$ can be written as

$$
w(t)=u(t)\left[C-\sum_{k=-\infty}^{+\infty} \widehat{r}(t-k W)\right] .
$$

Substituting (42) in (41) yields

$$
P_{0} \hat{f}(t) * u(t)=u(t)\left[C-\sum_{k=-\infty}^{-1} \widehat{r}(t-k W)\right] .
$$

The first term is the constant-limiting value the step response converges to when $t$ goes to infinity. Again if the impulse response is time-limited, the infinite sum can be reduced from $-K-1$ to -1 . The Laplace transform of the signal equals

$$
\hat{F}(s) \frac{P_{0}}{s}=\hat{F}(0) \frac{P_{0}}{s}-\widehat{R}(s) \sum_{k=-K-1}^{-1} e^{-k W s} .
$$

This can be rewritten as

$$
\widehat{R}(s) \sum_{k=-K-1}^{-1} e^{-k W s}=P_{0} \frac{\hat{F}(0)-\hat{F}(s)}{s} .
$$

Comparison of the latter to (21) shows that the reconstructed signal is nothing else than the smoothed negative-step response of the fiber plant. This proves that one can derive the negativestep response from any pulse response, on the correct preassumption that one knows the pulsewidth $W$, even if the first part of the transient is not available. The conversion is illustrated in Fig. 5.

This possibility enables an OTDR unit to combine reflections from transmitted pulses with different widths, as opposed to current state-of-the-art instruments that assume a constant pulsewidth. This makes the system very suitable for nonintrusive monitoring of burst-mode fiber networks because any burst that is transmitted by a fiber endpoint can be used to further characterize the smoothed fiber step response. This method is purely passive and data traffic is not congested at all. The biggest drawback of this nonintrusive method is the additional modulation noise that may lower the SNR considerably.

\section{PULSE OTDR}

Conventionally, OTDR instruments excite the fiber under test with a pulse, as modeled by (4). It is assumed that no

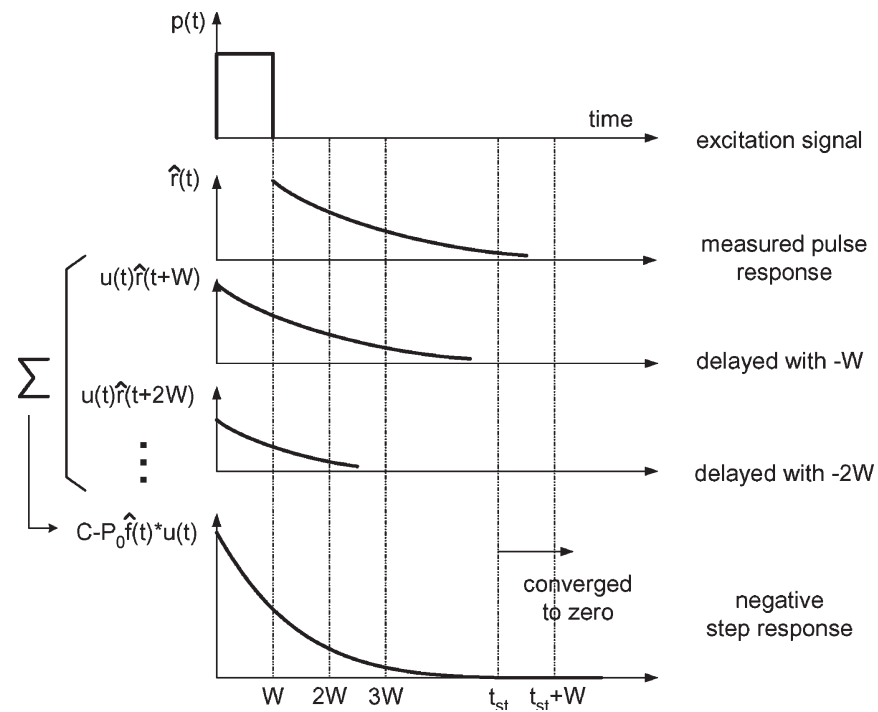

Fig. 5. Illustration of the transformation of the measured-pulse response into the negative-step response. The negative-step response starts from the constant limiting value of the step response at $t=0$ and becomes zero after $t_{\mathrm{st}}$ in case the fiber has finite length.

postprocessing is performed, so the function $G(s)$ from Fig. 2 is identically equal to one.

A distinction can be made between two operating modes. In case the pulsewidth $W$ is long enough, the RX with bandwidth $f_{c}$ has adequate time to respond to the pulse. If not, the $\mathrm{RX}$ reacts as if it was exited with an impulse with energy proportional to $W^{2}$. Classic OTDR instruments normally only operate in the first mode, because the fiber impulse response is measured instead of the pulse response and the reflectivity of certain discrete reflectors cannot be measured with good accuracy.

\section{A. Noise Analysis}

The output noise spectrum $S_{o}(f)$ of the RX is constant and equal to $S_{n 1}(f)$. The integrated noise is not infinite because of the limited bandwidth of the optical front end but a fixed constant.

$$
N=N 1=\int_{-\infty}^{+\infty} S_{n 1}(f) d f
$$

\section{B. Fresnel Reflections}

From (4) and (11), it follows that the expression for the measured reflection signal caused by a Fresnel reflection signal equals

$$
R(f)=R f_{\text {att }} V(f) P(f) e^{-j 2 \pi f \tau_{f r}}
$$

with

$$
f_{\text {att }}=e^{-2 \beta D} \text { with } \tau_{\text {fr }}=\frac{2 D}{\nu} .
$$


To calculate the signal power of the reflection, the inverse Fourier transform of the reflection signal to the power of two is evaluated

$$
|r(t)|^{2}=\left|\int_{-\infty}^{+\infty} R(f) e^{j 2 \pi f t} d f\right|^{2} .
$$

The reflection signal is a time delayed and scaled pulse; therefore, the maximum signal reflection occurs at $t=\tau_{\text {fr }}+W / 2$. Because $W \gg 1 / f_{c}, V(f)$ is approximated as $V(0)$

$$
\begin{aligned}
S & \cong\left[R f_{\text {att }} P_{0} V(0)\right]^{2}\left|\int_{-\infty}^{+\infty} \frac{1-e^{-j 2 \pi f W}}{j 2 \pi f} e^{j \pi f W} d f\right|^{2} \\
& =\left[R f_{\text {att }} P_{0} V(0)\right]^{2}
\end{aligned}
$$

The signal power from Fresnel reflections is independent of the pulsewidth $W$ as similar to the noise. This proves that exciting with a wider pulse does not improve the SNR of the measured optical reflections. It is even detrimental as wider reflection pulses overlap sooner, decreasing the OTDR distance resolution.

\section{Backscatter Reflections}

The reflection signal caused to a pulse $P_{0} p(0, W)$ with the width $W$ by backscatter from an infinite section of fiber can be deduced from (4) and (6) as

$$
R(f)=P(f) V(f) \frac{\eta \tau_{\mathrm{bs}}}{1+j 2 \pi f \tau_{\mathrm{bs}}} .
$$

The maximum signal power occurs at $t=W$. Again $V(f)$ is approximated as $V(0)$

$$
\begin{aligned}
|r(t)|^{2} & =\left|\int_{-\infty}^{+\infty} P_{0} \frac{1-e^{-j 2 \pi f W}}{j 2 \pi f} \frac{\eta \tau_{\mathrm{bs}} V(f)}{1+j 2 \pi f \tau_{\mathrm{bs}}} e^{j 2 \pi f t} d f\right|^{2} \\
S & \cong\left[P_{0} \eta \tau_{\mathrm{bs}} V(0)\right]^{2}\left|\int_{-\infty}^{+\infty} \frac{\sin (\pi f W)}{\pi f} \frac{e^{j \pi f W}}{1+j 2 \pi f \tau_{\mathrm{bs}}} d f\right|^{2} \\
& =\left[P_{0} \eta \tau_{\mathrm{bs}} V(0)\right]^{2}\left|1-e^{\frac{-W}{\tau_{\mathrm{bs}}}}\right|^{2} \\
& \cong\left[P_{0} \eta W V(0)\right]^{2} .
\end{aligned}
$$

For the last approximation, it was assumed that the backscatter time constant $\tau_{\text {bs }}$ is much larger than the excitation pulsewidth $W$, which under normal operating conditions can be assumed to be correct. It shows that the output SNR of backscatter signals, measured with a bigger pulsewidth $W$, will improve proportionally to $W^{2}$. Due to the low dynamic range of embedded OTDRs, it is necessary to operate with large pulsewidths in order to measure the backscatter, at the expense of decreased OTDR measurement resolution.

\section{Negative-Step OTDR}

In this section, the possibilities of using the negative-step response itself to categorize the channel properties are investigated and compared to the traditional approach described above.

\section{A. Noise Analysis}

If no postprocessing is needed, which once again results in a postprocessing function $G(s)$ identical to one, therefore, the integrated noise is constant and given by (46).

\section{B. Fresnel Reflections}

In case of the Fresnel reflection described by (11), from (21), it can be found that the reflection signal equals

$$
R(s)=\frac{P_{0}}{s}\left[R f_{\mathrm{att}} V(0)-R f_{\mathrm{att}} e^{-s \tau_{\mathrm{fr}}} V(s)\right] .
$$

Using the relationships

$$
\begin{aligned}
q(0) & =\lim _{s \rightarrow \infty} s Q(s) \\
\lim _{t \rightarrow+\infty} q(t) & =\left.s Q(s)\right|_{s=0}
\end{aligned}
$$

it can be found that

$$
\begin{aligned}
r(0) & =P_{0} R f_{\text {att }} V(0) \\
\lim _{t \rightarrow+\infty} r(t) & =0 .
\end{aligned}
$$

Therefore, in contrast to the pulse excitation, the negative-step excitation results in a down step in the reflection at the moment of the reflector. The step size is

$$
S=|r(0)|^{2}=\left[R f_{\text {att }} P_{0} V(0)\right]^{2}
$$

which is exactly the same number as in (50). Because the noise is also identical, the resulting SNR is identical when compared to pulse OTDR, the only difference is the type of event that occurs. This will be discussed further.

\section{Backscatter Reflections}

In case of backscatter, using (6) yields the measured reflection signal

$$
R(s)=\frac{P_{0}}{s}\left[\eta \tau_{\mathrm{bs}} V(0)-\frac{\eta \tau_{\mathrm{bs}}}{1+s \tau_{\mathrm{bs}}} V(s)\right] .
$$

Using the theorems of (55), in case the RX has a low-pass transfer function, the starting value becomes

$$
r(0)=P_{0} \eta \tau_{\mathrm{bs}} V(0)
$$

which leads to a maximal signal energy at $t=0$ that is equal to

$$
S=|r(0)|^{2}=\left[P_{0} \eta \tau_{\mathrm{bs}} V(0)\right]^{2} .
$$

Both excitations result in an exponentially decaying function with the same time constant. Comparing (60) to (53) shows that 
the big difference is that both curves are scaled with a factor $\tau_{\text {bs }} / W$. The backscatter time constant depends on the fiber attenuation, but for a typical ITU-T G.652 compliant single-mode fiber, it ranges around $60 \mu \mathrm{s}$. Because the output noise floor for both measurements is the same, the measurement accuracy for backscatter is, therefore, significantly better. In fact, negativestep OTDR increases the excitation pulsewidth to its maximum, while still maintaining distance resolution required for close-by Fresnel reflectors. In case the fiber section is finite, the SNR improvement still applies but decays gradually when the end of the fiber is approached. Only in the last section with duration $W$ of the reflection, the SNR becomes identical to the SNR of the pulse response. Maximization of the reflection signal is also positive, because it makes the measurement more prone against interference.

\section{Event Detection}

In case an embedded OTDR is monitoring a fiber plant, measuring the optical reflections alone is not enough to ratify the connected optical fiber. Postprocessing the acquired traces is required to extract relevant events that may reveal certain network discrepancies. There is a difference between reflective and nonreflective events. The first is only caused by backscatter reflections that no longer decay exponentially. The second also involves Fresnel reflectances that have higher bandwidth than the backscatter.

The bandwidth of the embedded OTDR RX is selected according to the required spatial resolution. Given a 5-MHz RX bandwidth, the reflection after a pulse response decays $5 \mathrm{~dB}$ at $7 \mathrm{~m}$ behind the pulse reflection [19]. This is adequate to test the compliance of PONs and identify the exact location of certain problems. The sensitivity of an embedded OTDR depends on the responsivity of the reflection detection and the sensitivity of the TIA. Given the 0.2-A/W responsivity from [11] and a typical TIA input referred noise of 1.5-nA rms, the sensitivity of the embedded OTDR equals $-51.2 \mathrm{dBm}$, which can be improved to $-74.7 \mathrm{dBm}$ after 50000 averages. In the following, a few simulations are shown, which illustrate and compare reflective- and nonreflective-event detection with pulse and negative-step OTDR. It is shown that the latter has significant advantages over the first when applied to an embedded OTDR. The average optical excitation power equals $3 \mathrm{dBm}$. This is the maximum allowed by GPON, but given the length of the fiber under test, this big power would certainly be arbitrated. For pulse OTDR, the excitation pulsewidth is adjusted to the RX bandwidth or vice-versa. Given the 5-MHz bandwidth, a 200-ns excitation pulse is chosen.

\section{A. Reflective Events}

In case the fiber under test contains a Fresnel reflector, the optical reflection is identical to the excitation function. For pulse OTDR, a pulse will be reflected containing a rising and falling edge, yet, in case of negative-step OTDR, it results in a single down step. With the same RX bandwidth for both methods, this can prove beneficial to the resulting spatial resolution. In illustration, a network was simulated, which contains two
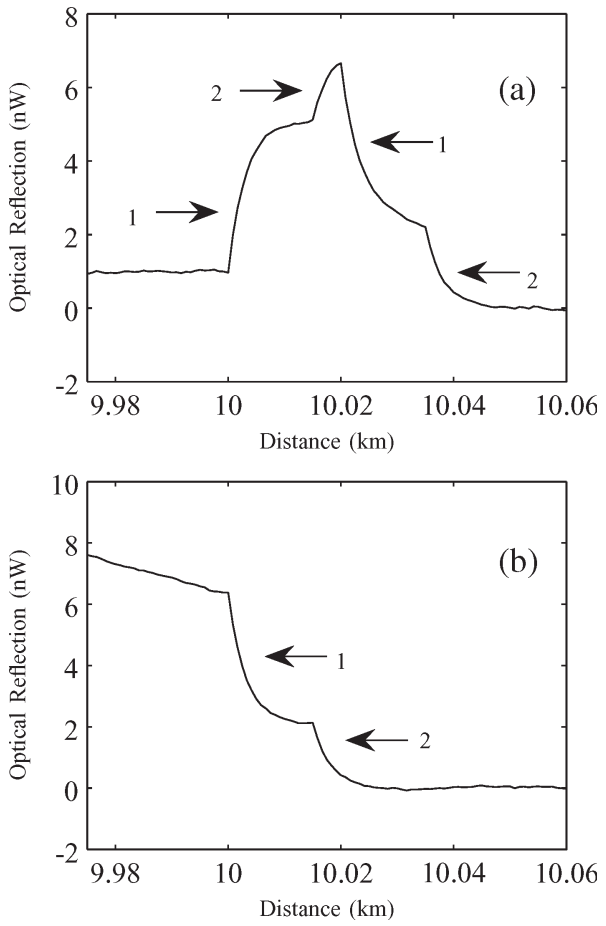

Fig. 6. Simulation of the optical reflection measured by an embedded OTDR RX in case the fiber is excited (a) with a 200-ns pulse or (b) with a negative step. The closeup reveals two discrete reflectors at distance $15 \mathrm{~m}$ with the second half the size of the first. The artefacts caused by events 1 and 2 are indicated.

close-by Fresnel reflectors of size -50 and $-53 \mathrm{~dB}$ at $10 \mathrm{~km}$ and $15 \mathrm{~m}$ farther, respectively.

Fig. 6(a) shows a close-up of the reflection as measured by pulse OTDR. It shows that both reflection pulses overlap with each other, which results in a strange waveform with several rising and falling edges. A human operator would resort to a smaller excitation pulse to get rid of the overlap. An embedded OTDR instrument has to monitor the events automatically. Detecting and interpreting overlapped pulses is hard and reducing the pulsewidth is even impossible due to the fixed receiver bandwidth. Turning to the negative-step response from Fig. 6(b), it shows that both Fresnel reflectances are separated in time and can easily be distinguished from each other. Because the pulses do not overlap, the two different reflectors at 15-m distance can be distinguished and measured with better SNR because a smaller excitation pulse would require a larger receiver bandwidth and a higher noise floor.

\section{B. Nonreflective Events}

For nonreflective events like splice losses, more postprocessing is required to extract the relevant parameters like the splice loss. In the illustration, simulations were made on a cascade of 10 and $8 \mathrm{~km}$ of ITU-T G.652 compliant sections of singlemode fiber. The reflection system contains no discrete reflectors, but there is a $0.3-\mathrm{dB}$ splice loss between both sections that should be identified. The simulated fiber for both sections is Draka Comteq Bendbright single-mode fiber with a relatively low backscatter constant at $1310 \mathrm{~nm}$ of $-79.4 \mathrm{~dB}$ for a 1-ns excitation pulse. A 200-ns excitation pulse, therefore, leads to backscatter at the origin of $3-79.4+10 \log (200)$ or 


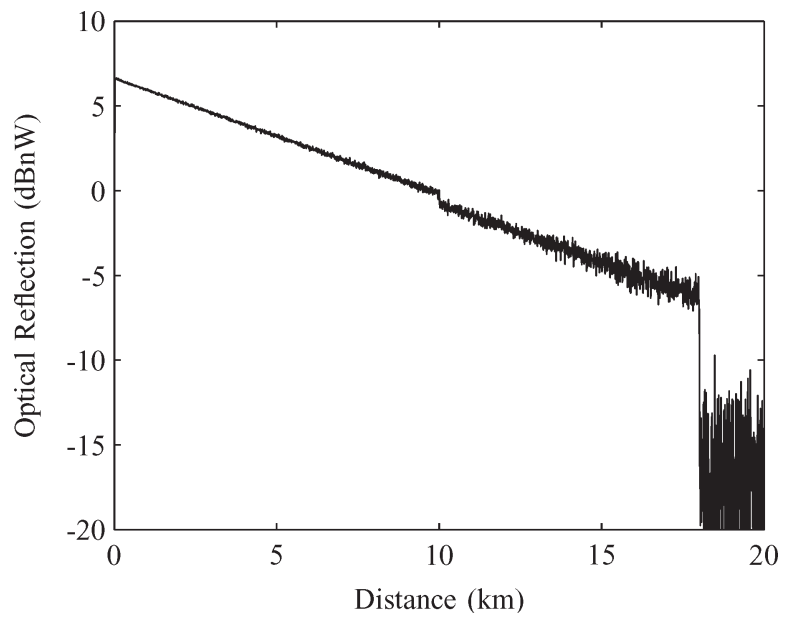

Fig. 7. Simulation of the 200-ns pulse response measured by an embedded OTDR RX with 50000 averages. The small 0.3-dB splice loss can be noticed, but it is hard to detect and measure the event with adequate accuracy.

$-53.4 \mathrm{dBm}$, and after $10 \mathrm{~km}$, the backscatter power has even dropped by $6.8 \mathrm{~dB}$ to an ample $-60.2 \mathrm{dBm}$, which is barely larger than the noise floor even after averaging 50000 times. The resulting pulse response from Fig. 7 illustrates that the nonreflective event at $10 \mathrm{~km}$ can perhaps be recognized, but estimating the splice loss with good accuracy certainly is impossible. Therefore, using an embedded OTDR, normal-pulse OTDR operation mode does not lead to accurate results when detecting nonreflective events.

The bandwidth of the backscatter process is substantially lower than the 5-MHz receiver bandwidth required for good spatial resolution. Therefore, reducing the bandwidth of the backscatter reflections can improve the SNR when nonreflective events are estimated. During a period $L$ that is substantially lower than the backscatter time constant $\tau_{\text {bs }}$ from (6), the exponentially decaying backscatter reflection can be approximated by a linear function, using the linear-regression method described in the Appendix. It is assumed that the reflection signal is sampled with sample period $T_{s}$, in such a way that subsequent noise samples are independent with standard deviation $\sigma$. This means that the sampling rate is approximately twice the RX bandwidth. In our case, the sampling rate was chosen $12 \mathrm{MSps}$ as the filter shape is not rectangular, so the sampling period $T_{s}$ is $83.3 \mathrm{~ns}$. $N$ samples are taken during the regression period $L$, meaning that $L>(N-1) T_{s}$. Given $N$, the correlation function (77) estimates the average of the pulse response. Under the condition that $\tau_{\mathrm{bs}} \gg L$, the average is a good estimate of the backscatter and reduces the noise standard deviation according to $(75)$.

Deriving the negative-step response and multiplying it by -1 results in the impulse response of the system. Therefore, a similar linear-regression strategy can be applied to negativestep OTDR to improve the measurement accuracy by estimating the slope instead of the average and linear regressing with sequence (78). Using this methodology, the variance on the measurement is reduced according to (76). The scaled-impulse response with a factor equal to the pulsewidth $W$ is the same as the linear-regressed pulse response when no Fresnel reflectors

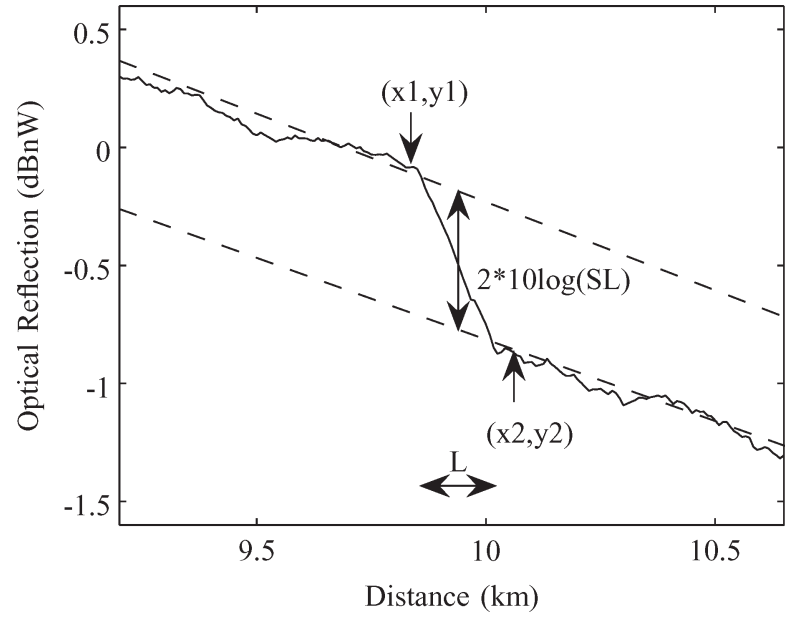

Fig. 8. Explanatory figure about estimating the splice loss SL of the nonreflective event. Because the same fiber with the same $\eta$ and $\tau_{\mathrm{bs}}$ was used, the backscatter degrades with the same slope and the splice estimation does not involve the changing $\eta$. The figure also shows the influence of the regression period $L$.

occur, which is the case for a nonreflective event. Both pulse and negative-step reflections can be used to estimate the splice loss after linear regression.

Because the signal powers are the same, the ratio of the SNR of linear-regressed negative-step OTDR to the linear-regressed pulse OTDR can be found by comparing the noise variances after linear regression, taking the scaling factor $W$ into account. From (75) and (76), we find

$$
W^{2} \frac{12 \sigma^{2}}{T_{s}^{2} N(N+1)(N-1)} \frac{N}{\sigma^{2}}=\frac{12 W^{2}}{\left(N^{2}-1\right) T_{s}^{2}} .
$$

In case $N$ is big enough, the OTDR signal resulting from negative-step OTDR has better SNR than in the case of the pulse OTDR. The threshold value equals

$$
N_{\mathrm{th}}=\sqrt{12 \frac{W^{2}}{T_{s}^{2}}+1}
$$

In the presented setup, this results in 8.3 samples, leading to a regression period $L$ larger than $0.61 \mu$ s, which can be recalculated into a distance of $61 \mathrm{~m}$. The burden on distance resolution is not very high under these conditions, but it can increase the measurement accuracy of nonreflective events a lot. It should be noted that increasing sampling rate does not improve accuracy a lot, because the preassumption that subsequent noise samples are independent then no longer holds.

1) Splice-Loss Estimation: Still, further processing is required after linear regression to estimate the splice loss. In Fig. 8, the measurement points $\left(x_{1}, y_{1}\right)$ and $\left(x_{2}, y_{2}\right)$ are placed right before and after the nonreflective event. In here, the ordinate $x$ represents time rather than the distance and the measurements are expressed in linear scale. It is clear that the difference $\Delta x=x_{2}-x_{1}$ should be bigger than the regression period $L$. Given $\eta_{1}$ and $\eta_{2}$, the backscatter constant of the first and second section, and $\tau_{\mathrm{bs} 1}$ and $\tau_{\mathrm{bs} 2}$, their respective 
backscatter time constant, the estimation of the splice loss SL equals

$$
\mathrm{SL}^{2}=\frac{\eta_{1} y_{1}}{\eta_{2} y_{2}} e^{-0.5 \Delta x\left(1 / \tau_{\mathrm{bs} 2}+1 / \tau_{\mathrm{bs} 1}\right)} .
$$

It is assumed that all the backscatter parameters are known or can be estimated from the rest of the measurement with adequate accuracy. Inaccuracies on such estimations are out of scope of this derivation.

After linear regression, both pulse and the scaled negativestep OTDR result in the same signal power for nonreflective events, yet, the noise powers differ. Because the linearregression processes (77) and (78) are linear, both the estimation of the average and slope transform the optical front-end noise $S_{n 0}(f)$ to colored noise, which has a normal distribution if sampled. Therefore, it can be assumed for both methods that the measurements $y_{1}$ and $y_{2}$ have a normal distribution with variance as predicted by (75) and (76). If the SNR on $y_{1}$ and $y_{2}$ is high, the distribution of the ratio of two normally distributed variables is approximately, also normally, distributed variable. In case the SNR on the measurement points is higher, the variance on the ratio will also be smaller. Therefore, a better approximation on the splice loss can be made with negativestep OTDR in case the regression period is chosen long enough.

\section{Benefits OF NEGATIVE-STEP OTDR}

In Section III, a methodology was introduced that uses reflections from data burst to characterize the fiber under test. Any data burst is transmitted by the optical communications node can improve knowledge of the connecting fiber. This nonintrusive detection allows the gathering of many measurements in the background of an operating optical network, which can all be averaged to reduce the noise floor. Traditional techniques require the data traffic to be halted in order to perform OTDR.

The tradeoff between spatial resolution and backscatter SNR is a well-known for pulse OTDR. The backscatter reflections, which decay exponentially, size with the width of the excitation pulse. Therefore, a wider pulse is preferred when measuring small backscatter, but this reduces the spatial resolution because the dead zones also depend on the pulsewidth $W$, with the result that close-by events no longer can be distinguished from each other. The negative-step response solves this problem as the Fresnel reflector now results in a single downward step instead of a pulse that can be mangled with other reflected pulses while maximizing the backscatter reflections. The dead zone only depends on the rise or fall time of the step response, which is caused by the RX bandwidth only and, therefore, considerably lower.

The excitation power and sensitivity of an embedded OTDR are low. Negative-step excitation does not change the excitation power nor does it lower the RX noise, yet, it maximizes the backscatter-reflection signal power, which improves its tolerance to interference. Because the RX bandwidth is fixed by the required spatial resolution, pulse OTDR results in noisy measurement traces. Negative-step OTDR results in the same SNR for Fresnel reflectors but contains improvement for nonreflective events. Because the bandwidth of backscatter is appreciably lower, linear regression over a long time-span is allowed which allows for substantial SNR improvement of negative-step OTDR with respect to pulse OTDR. Moreover, the fact that this linear regression is pure postprocessing makes it a very powerful method. The regression period can be chosen long or short, or a combination of both for different events occurring in the same OTDR trace.

\section{CONCLUSION}

A novel method for performing OTDR measurements was proposed, which can characterize the status of an optical communication channel nonintrusively by measuring the optical echoes caused by transmitted data bursts and processing them into a standardized format. This negative-step OTDR was validated against the conventional pulsed OTDR. From theory and simulations, the benefits of negative-step OTDR with respect to pulse OTDR were demonstrated.

\section{APPENDIX \\ LINEAR REGRESSION}

The linear-regression method is a statistical procedure for estimating the conditional expected value of a dependent variable $Y$ given the values of an independent variable $X$ when the relationship between the variables can be described with a linear model. A linear-regression equation can be written as

$$
Y=\alpha_{0}+\alpha_{1} X+\epsilon
$$

where $\alpha_{1}$ is the slope of the regression line and $\alpha_{0}$ is the $Y$-intercept of the regression line. The term $\epsilon$ represents the unpredicted or unexplained variation in the dependent variable; it is called the error whether it is really a measurement error or not. The error term is conventionally assumed to have expected value equal to zero; otherwise, it can be absorbed into $\alpha_{0}$

$$
E[Y \mid X]=\alpha_{0}+\alpha_{1} X
$$

We can estimate the unobservable values $a_{0}$ and $a_{1}$ of the parameters $\alpha_{0}$ and $\alpha_{1}$ by the method of least squares, which consists of finding the values of $a_{0}$ and $a_{1}$ that minimize the sum of squares of the residuals

$$
e_{n}=y_{n}-\left(a_{0}+a_{1} x_{n}\right) \text {. }
$$

As $X$ is an independent variable, $x_{n}$ represents a set of $N$ known values ranging from $x_{0}$ to $x_{N-1}$, whereas $y_{n}$ corresponds to the accordingly observed values. Using the method of least squares, we can derive $a_{0}$ and $a_{1}$, the estimates of $\alpha_{0}$ and $\alpha_{1}$

$$
\begin{aligned}
& a_{0}=\frac{\sum_{n} y_{n}\left[\sum_{n} x_{n}^{2}-N^{2} \bar{x}^{2}\right]}{N \sum_{n} x_{n}^{2}-N^{2} \bar{x}^{2}} \\
& a_{1}=\frac{\sum_{n}\left(x_{n}-\bar{x}\right)\left(y_{n}-\bar{y}\right)}{\sum_{n}\left(x_{n}-\bar{x}\right)^{2}}
\end{aligned}
$$


where $\bar{x}$ and $\bar{y}$ are the mean of $x_{n}$ and $y_{n}$, respectively. The variance on $a_{0}$ and $a_{1}$ can be derived by considering them as a linear combination of $N$ mutual-uncorrelated samples $y_{n}$, with variance $\sigma^{2}$

$$
\begin{aligned}
& \operatorname{Var}\left[a_{0}\right]=\frac{\sigma^{2}}{N}\left(1+\frac{\bar{x}^{2}}{s_{x}^{2}}\right) \\
& \operatorname{Var}\left[a_{1}\right]=\frac{\sigma^{2}}{N s_{x}^{2}}
\end{aligned}
$$

with $s_{x}^{2}$, the sample standard deviation

$$
s_{x}^{2}=\frac{1}{N} \sum_{n}\left(x_{n}-\bar{x}\right)^{2} .
$$

This technique can be applied to data coming from a sampling system that digitizes continuous signals $y(t)$ to a batch of samples $y[n]=y_{n}=y\left(n T_{s}+p\right)$, with $T_{s}$ as the sampling period and $p$ as a real-valued constant. From (69), it shows that the variance on $a_{0}$ depends on $\bar{x}$ and the smallest variance is observed when this mean equals zero. Neglecting the effects of clock jitter, we can choose $x_{n}$ as equidistant time stamps at distance $T_{s}$ for the $N$ samples $y_{n}$ with parameter $n$ delimited by boundaries $[0, N-1]$. Choosing

$$
x_{n}=T_{s}\left(n-\frac{N-1}{2}\right)
$$

results in $\bar{x}$ equal to zero, and this makes the regression insensitive to the absolute offset on $x$. We find

$$
\begin{aligned}
a_{0} & =\frac{\sum_{n} y[n]}{N} \\
a_{1} & =\frac{\sum_{n} x_{n} y[n]}{\sum_{n} x_{n}^{2}} .
\end{aligned}
$$

The latter holds because $\sum_{n} x_{n} \bar{y}$ is equal to zero. The variance on $a_{0}$ and $a_{1}$ are now

$$
\begin{aligned}
& \operatorname{Var}\left[a_{0}\right]=\frac{\sigma^{2}}{N} \\
& \operatorname{Var}\left[a_{1}\right]=\frac{12 \sigma^{2}}{T_{s}^{2} N(N+1)(N-1)} .
\end{aligned}
$$

It can be noticed that both (73) and (74) can be considered as the correlation of the digitized samples $y[n]$ with a fixed sequence that can be calculated from the known values $N$ and $T_{s}$. To obtain $a_{0}$, we correlate $y_{n}$ with a constant sequence of value $1 / N$, for $a_{1}$ with a linearly varying sequence

$$
\begin{aligned}
& c_{0}[n]=1 / N \\
& c_{1}[n]=\frac{12}{T_{s}} \frac{n-1 / 2(N-1)}{N(N+1)(N-1)} .
\end{aligned}
$$

We now can obtain the time-varying correlations $a_{0}[n]$ and $a_{1}[n]$ as

$$
\begin{aligned}
& a_{0}[n]=c_{0}[n] \otimes y[n] \\
& a_{1}[n]=c_{1}[n] \otimes y[n]
\end{aligned}
$$

where $\otimes$ is the correlation operator. In here, $a_{0}$ is an estimation of the average of the signal and $a_{1}$ is an estimation of the signal derivative for the investigated batch of samples.

\section{ACKNOWLEDGMENT}

This work results from an internal research project performed at INTEC_design. The authors would like to thank the University of Ghent and IMEC/Intec for offering the opportunity to perform this research.

\section{REFERENCES}

[1] D. Kilper, R. Bach, D. Blumenthal, D. Einstein, T. Landolsi, L. Ostar, M. Preiss, and A. Willner, "Optical performance monitoring," J. Lightw. Technol., vol. 22, no. 1, pp. 294-304, Jan. 2004.

[2] ITU-T Rec. L.25: Optical Fibre Cable Network Maintenance, 1996.

[3] R. Asthana and Y. N. Singh, "Protection and restoration in optical networks," IETE J. Res., vol. 50, no. 5, pp. 319-329, Sep. 2004.

[4] M. P. Gold, "Design of a long-range single-mode OTDR," J. Lightw. Technol., vol. LT-3, no. 1, pp. 39-46, Feb. 1985.

[5] H. Lee, Y. Nam, D. Lee, H. Chung, and K. Kim, "Demonstration of a low-cost 1625-nm OTDR monitoring for 350-km WDM networks with semiconductor optical amplifiers," IEEE Photon. Technol. Lett., vol. 17, no. 4, pp. 852-854, Apr. 2005.

[6] H. Schmuck, J. Hehmann, and T. Pfeiffer, "Erhohte zuverlassigkeit von optischen zugangsnetzen durch integrierte faseruberwachung," Köln, Germany, ITG-Fachbericht no. 185, 2004.

[7] H. Schmuck, J. Hehmann, M. Straub, and T. Pfeiffer, "Embedded OTDR techniques for cost-efficient fibre monitoring in optical access networks," in Proc. 32th ECOC, Cannes, France, 2006, vol. 1, pp. 49-50.

[8] D. K. Mynbaev, "Optical access: Networks and components (overview)," Proc. SPIE-Int. Soc. Opt. Eng., vol. 5598, pp. 81-92, Oct. 2004.

[9] J. Bauwelinck, W. Chen, D. Verhulst, Y. Martens, P. Ossieur, X.-Z. Qiu, and J. Vandewege, "A high-resolution burst-mode laser transmitter with fast and accurate level monitoring for $1.25 \mathrm{gb} / \mathrm{s}$ upstream GPONs," IEEE J. Solid-State Circuits, vol. 40, no. 6, pp. 1322-1330, Jun. 2005.

[10] ITU-T Rec. G.984.2: Gigabit-Capable Passive Optical Networks (GPON): Physical Media Dependent (PMD) Layer Specification, 2003.

[11] G. Keeler, D. Serkland, K. Geib, and G. Peake, "In situ OTDR for lowcost optical networks using singlemode $850 \mathrm{~nm}$ VCSEL," Electron. Lett., vol. 41, no. 14, pp. 819-820, Jul. 2005.

[12] J. Vandewege, B. De Mulder, W. Chen, and X.-Z. Qiu, "Circuit, system and method for monitoring an optical fibre network," Oct. 7, 2005. EPO and U.S. patents pending.

[13] H. Takasugi, N. Tomita, J. Nakano, and N. Atobe, "Design of a 1.65- $\mu \mathrm{m}$-band optical time-domain reflectometer," J. Lightw. Technol., vol. 11 , no. 11, pp. 1743-1748, Nov. 1993.

[14] A. H. Hartog and M. P. Gold, "On the theory of backscattering in single-mode fibers," J. Lightw. Technol., vol. LT-2, no. 2, pp. 76-82, Apr. 1984.

[15] F. P. Kapron, B. P. Adams, E. A. Thomas, and J. W. Peters, "Fiber-optic reflection measurements using OCWR and OTDR techniques," J. Lightw. Technol., vol. 7, no. 8, pp. 1234-1241, Aug. 1989.

[16] J. L. Hullett and T. V. Muoi, "A feedback receive amplifier for optical transmission systems," IEEE Trans. Commun., vol. COM-24, no. 10, pp. 1180-1185, Oct. 1976.

[17] M. Nazarathy, S. A. Newton, R. P. Giffard, D. S. Moberly, F. Sischka, W. R. J. Trutna, and S. Foster, "Real-time long range complementary correlation optical time domain reflectometer," J. Lightw. Technol., vol. 7 , no. 1, pp. 24-38, Jan. 1989.

[18] M. Tateda and T. Horiguchi, "Advances in optical time domain reflectometry," J. Lightw. Technol., vol. 7, no. 8, pp. 1217-1224, Aug. 1989.

[19] D. Anderson, L. Johnson, and F. G. Bell, Troubleshooting Optical Fiber Networks, First Edition: Understanding and Using Optical Time-Domain Reflectometers. New York: Academic, 2004. 


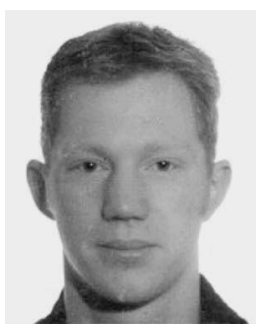

Bert De Mulder (S'06) was born in Sint-Niklaas, Belgium, in 1979. He received the Engineering degree in applied electronics from Ghent University, Ghent, Belgium, in 2001, where he is currently working toward the Ph.D. degree.

Since 2001, he has been working at the INTEC design Laboratory, Ghent University, on various projects regarding the design of instrumentation. His research is currently focused on the development of monitoring hardware for nonintrusive embedded optical time-domain reflectometer.

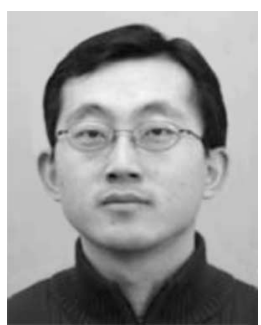

Wei Chen (S'06) was born in YanZhou, China, in 1974. He received the Bachelor's degree in electrical engineering and the Master's degree in communication and signal processing from Beijing University of Aeronautics and Astronautics, Beijing, China.

From 2000 to 2002, he worked in Cellon China Group for 3-G wireless-communication research project. Since 2002, he has been working as a Ph.D. candidate in the INTEC_design Laboratory, Ghent University, Ghent, Belgium. His research is currently focused on reconfigurable test platforms for nonintrusive embedded optical time-domain reflectometer.

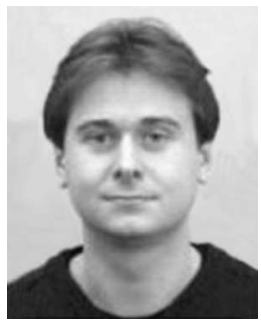

Johan Bauwelinck (M'05) was born in SintNiklaas, Belgium, in 1977. He received the Engineering degree in applied electronics and the Ph.D. degree in applied sciences, electronics from Ghent University, Ghent, Belgium, in 2000 and 2005, respectively.

He has been a Research Assistant in the INTEC design Laboratory, Ghent University, since 2000. His research is focused on analog-integrated-circuit design.

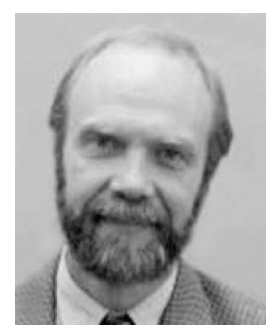

Jan Vandewege (M'96) was born in Gent, Belgium, in 1949. He received the degree in electronic engineering and the Ph.D. degree from Ghent University, Ghent, Belgium, in 1972 and 1978, respectively.

In 1985, he founded the INTEC_design Laboratory at Ghent University, to train Ph.D.-level electronic engineers in design of telecom and RF hardware and embedded software. He (co)authored 134 international publications and ten international patents in the field of telecommunication.

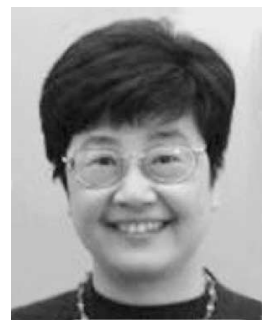

Xing-Zhi Qiu (M'97) received the Ph.D. degree in electronics engineering from Ghent University, Ghent, Belgium.

Since 1986, she has been with the INTEC_design Laboratory, Ghent University. She is currently managing high-speed mixed analog/digital chip and subsystem design. She accumulated 16 years of $R \& D$ experience in the field of hardware design for the physical layer, mostly developing advanced telecommunication systems and optical-access network demonstrators. She is author/coauthor of 70 international publications. 